\title{
Combination of chloroquine and GX15-070 (obatoclax) results in synergistic cytotoxicity against pancreatic cancer cells
}

\author{
GUAN WANG $^{1}$, SHAOHUA CHEN ${ }^{1}$, HOLLY EDWARDS $^{3,4}$, XINMING CUI $^{2}$, LI CUI $^{2}$ and YUBIN GE ${ }^{1,3,4}$ \\ ${ }^{1}$ National Engineering Laboratory of AIDS Vaccine, Key Laboratory for Molecular Enzymology and Engineering, \\ the Ministry of Education, School of Life Sciences, Jilin University; ${ }^{2}$ Key Laboratory of Pathobiology, \\ Ministry of Education, Norman Bethune College of Medicine, Jilin University, Changchun, P.R. China; \\ ${ }^{3}$ Department of Oncology, Wayne State University School of Medicine; ${ }^{4}$ Molecular Therapeutics Program, \\ Barbara Ann Karmanos Cancer Institute, Wayne State University School of Medicine, Detroit, MI, USA
}

Received July 9, 2014; Accepted August 18, 2014

DOI: $10.3892 /$ or.2014.3525

\begin{abstract}
Pancreatic cancer is an aggressive disease with a poor prognosis. Therefore, new treatment is urgently required. GX15-070 is a pan-Bcl-2 inhibitor which has shown promising antitumor activity in different malignancies. We previously demonstrated that clinically achievable concentrations of GX15-070 caused growth arrest in pancreatic cancer cell lines. However, they only induced minimal levels of apoptosis. We hypothesized that GX15-070 induced autophagy in pancreatic cancer cells which blocked apoptosis. In this study, we investigated the effects of GX15-070 on autophagy and the antitumor activities of the combination of GX15-070 and chloroquine (CQ), an autophagy inhibitor, in six pancreatic cancer cell lines. We found that GX15-070 treatment indeed induced autophagy in 5 of the 6 pancreatic cancer cell lines, reflected by the conversion of LC3B-I to LC3B-II and detection of autophagosomes by transmission electron microscopy. Furthermore, we found additive to synergistic antitumor interactions in all six cell lines by MTT assays. CQ significantly enhanced GX15-070-induced apoptosis in the cell line models, possibly due to downregulation of Bcl-2, Bcl-xL and Mcl-1 in the cells by the two agents. These results provide compelling evidence for the further development of the combination of GX15-070 and CQ in pancreatic cancer.
\end{abstract}

\section{Introduction}

Pancreatic cancer represents $<3 \%$ of all new cancer cases in the United States; however, it is the fourth leading cause of cancer-related mortality (1). With a 5-year survival rate of

Correspondence to: Dr Yubin Ge, Department of Oncology, Wayne State University School of Medicine, 110 East Warren Avenue, Detroit, MI 48201, USA

E-mail: gey@karmanos.org

Key words: chloroquine, GX15-070, pancreatic cancer, drug combination, autophagy $\sim 6 \%$, the prognosis of this disease is considerably poor (1). Although surgical resection offers the only chance for cure, the 5 -year survival rate is only $20-25 \%$ and $\sim 80-85 \%$ of patients present with advanced unresectable disease (2). In addition, the front-line gemcitabine-based therapy offers marginal efficacy, thus highlighting the need to develop new therapies for this aggressive disease.

Cancer cells employ several mechanisms of survival, including overexpression of prosurvival Bcl-2 family members, making them attractive targets for the treatment of numerous malignancies. Since $88 \%$ of invasive ductal carcinomas express Bcl-xL and Mcl-1, while 23\% express Bcl-2 (3), small molecule inhibitors that target these proteins could be promising therapeutic agents for the treatment of pancreatic cancer.

GX15-070 (obatoclax) is a pan-Bcl-2 inhibitor that has been demonstrated to directly induce apoptosis as well as non-apoptotic cell death in solid tumors and hematologic malignancies (4-8). In our most recent study, we demonstrated that clinically achievable concentrations of GX15-070 suppressed the growth of pancreatic cancer cell lines. However, they only induced minimal levels of apoptosis in the cells (9). We hypothesized that GX15-070 induced autophagy which prevented apoptosis in pancreatic cancer cells since Bcl-2 family proteins also play an important role in autophagy. Beclin 1 is a $\mathrm{Bcl}-2$ binding protein that regulates the initial steps of autophagy (10). Bcl-2, Mcl-1, and Bcl-xL have been shown to interact with Beclin 1, binding through the $\mathrm{BH} 3$ domain, and inhibit autophagy $(10,11)$. In line with this, treatment with Bcl-2 inhibitors, such as GX15-070, have been shown to induce autophagy in various malignancies (12-15). Although autophagy has been shown to be involved in tumor suppression as well as therapeutic resistance, in pancreatic cancer it has been demonstrated to play a cytoprotective role against anticancer drugs $(16,17)$. Therefore, combining an autophagy inhibitor with GX15-070 may enhance GX15-070induced apoptosis in pancreatic cancer cells.

In the present study, we investigated the combination of GX15-070 and the autophagy inhibitor chloroquine (CQ) in pancreatic cancer cell lines. GX15-070 treatment alone induced autophagy in a dose-dependent manner. Addition of CQ resulted in additive to synergistic growth inhibition as well 
as enhanced apoptosis. These results suggest that combined GX15-070 and CQ might be a promising treatment for pancreatic cancer.

\section{Materials and methods}

Chemicals. GX15-070 (Obatoclax) was purchased from Selleck Chemicals LLC (Houston, TX, USA). CQ was purchased from Sigma-Aldrich (St. Louis, MO, USA).

Cell culture. The AsPC-1, BxPC-3, CFPAC-1, HPAC, MIA $\mathrm{PaCa}-2$ and PANC-1 human pancreatic cancer cell lines were purchased from the American Type Culture Collection (ATCC; Manassas, VA, USA). The HPAC, MIA PaCa-2 and PANC-1 cell lines were cultured in Dulbecco's modified Eagle's medium (DMEM; Life Technologies, Carlsbad, CA, USA); AsPC-1 and BxPC-3 were cultured in RPMI-1640 medium (Life Technologies); and CFPAC-1 in Iscove's modified Dulbecco's medium (IMDM; Life Technologies) with $10 \%$ heat-inactivated fetal bovine serum (FBS; Hyclone Labs, Logan, UT, USA) plus $100 \mathrm{U} / \mathrm{ml}$ penicillin and $100 \mu \mathrm{g} / \mathrm{ml}$ streptomycin in a $37^{\circ} \mathrm{C}$ humidified atmosphere containing 5\% $\mathrm{CO}_{2} / 95 \%$ air. Cell lines were authenticated by the University of Arizona Genetics Core Facility (Tucson, AZ, USA).

Western blot analysis. Whole cell lysates from AsPC-1, BxPC-3, CFPAC-1, HPAC, MIA PaCa-2 or PANC-1 cells treated with vehicle control or drugs for $72 \mathrm{~h}$ were subjected to SDS-polyacrylamide gel electrophoresis. Separated proteins were electrophoretically transferred to polyvinylidene difluoride (PVDF) membranes (Thermo Fisher Inc., Rockford, IL, USA) and immunoblotted with anti-LC3B, -PARP, -Mcl-1,-Bcl-2 , -Bcl-xL (Cell Signaling Technology, Beverly, MA, USA), or - $\beta$-actin (Sigma-Aldrich) antibody, as previously described (18). Immunoreactive proteins were visualized using the Odyssey Infrared Imaging System (Li-Cor, Lincoln, NE, USA), as described by the manufacturer. Western blot analyses were repeated at least three times and one representative image is shown.

Transmission electron microscopy (TEM). Briefly, PANC-1 cells were treated with vehicle control or $200 \mathrm{nM}$ GX15-070 for $72 \mathrm{~h}$. Then, the cells were trypsinized and washed with PBS twice. The cells were fixed with $2.5 \%$ glutaraldehyde in $0.1 \mathrm{M}$ sodium phosphate buffer ( $\mathrm{pH}$ 7.4). The samples were treated with $1 \%$ osmium acid, dehydrated in a graded series of ethanol, and embedded in Epon812 epoxy resin (SPI Supplies/Structure Probe, Inc., West Chester, PA, USA). Semithin sections were made using an LKB-III ultramicrotome (LKB Bromma, Stockholm, Sweden). Then ultrathin sections were made and picked up with naked 200 mesh copper grids. Grids were stained with uranyl acetate and lead citrate. The ultrathin sections were then observed using a Hitachi H-7650 transmission electron microscope (Hitachi High-Technologies Corporation, Tokyo, Japan) at $80 \mathrm{kV}$.

In vitro cytotoxicity assays. In vitro CQ or GX15-070 cytotoxicities were determined using 3-(4,5-dimethyl-2-thiazolyl)2,5-diphenyltetrazolium bromide (MTT) assays (SigmaAldrich), as previously described $(19,20)$. Briefly, cells were cultured in $100 \mu \mathrm{l}$ of complete medium in the presence of variable concentrations of GX15-070 or CQ alone or in combination. The cells were incubated at $37^{\circ} \mathrm{C}$ in 96 -well plates for $96 \mathrm{~h}$. MTT was added to a final concentration of $1 \mathrm{mM}$. After $4 \mathrm{~h}$, formazan crystals were dissolved by the addition of $100 \mu \mathrm{l}$ of $10 \%$ SDS in $10 \mathrm{mM} \mathrm{HCl}$. Optical densities were measured using a visible light microplate reader at $590 \mathrm{~nm} . \mathrm{IC}_{50}$ values were calculated as drug concentrations necessary to inhibit $50 \%$ growth compared to vehicle control-treated cells. The data are presented as mean values \pm standard errors from at least 3 independent experiments. The extent and direction of antitumor interactions were evaluated using CompuSyn software (ComboSyn, Inc., Paramus, NJ, USA). Drug interactions were quantified by determining the combination index $(\mathrm{CI})$, where $\mathrm{CI}<1, \mathrm{CI}=1$ and $\mathrm{CI}>1$ indicate synergistic, additive and antagonistic effects, respectively (21).

Apoptosis and cell cycle analysis. Cells were treated with CQ or GX15-070, alone or in combination for $72 \mathrm{~h}$. Then, cells were fixed with ice-cold $80 \%$ (v/v) ethanol for $24 \mathrm{~h}$, washed with PBS and resuspended in PBS containing propidium iodide $(50 \mu \mathrm{g} / \mathrm{ml})$, Triton X-100 $(0.1 \%, \mathrm{v} / \mathrm{v})$, and DNase-free RNase $(1 \mu \mathrm{g} / \mathrm{ml})$. DNA content was determined by flow cytometry analysis, as previously described (22). Cell cycle analysis was performed using Multicycle software (Phoenix Flow Systems, Inc., San Diego, CA, USA). Apoptotic events were recorded as $\mathrm{PI}^{+}$events with sub-G1 DNA content. The data are presented as mean values \pm standard errors from one representative experiment, which was repeated at least 3 independent times.

Statistical analysis. The data are expressed as the mean values \pm standard errors. The differences in $\mathrm{CQ} \mathrm{IC}_{50}$ between GX15-070-treated and vehicle control-treated cells and differences in cell apoptosis between CQ and GX15-070 combination-treated and GX15-070-treated cells were compared using the pairwise two-sample t-test. Statistical analyses were performed using GraphPad Prism 5.0.

\section{Results}

GX15-070 induces autophagy in pancreatic cancer cell lines. Bcl-2 family proteins play an important role in autophagy. Others have demonstrated that GX15-070, a pan-Bcl-2 inhibitor, induces autophagy in various cancer cell lines $(12-15,23)$. To begin to determine if GX15-070 induces autophagy in pancreatic cancer cells, we determined if GX15-070 treatment results in increased conversion of LC3B-I to LC3B-II. There was a dose-dependent increase in LC3B-II protein levels in all the cell lines tested, except AsPC-1 (Fig. 1A). To further confirm that GX15-070 induces autophagy in the pancreatic cancer cells, we treated PANC-1 cells with or without $200 \mathrm{nM}$ GX15-070 and examined autophagosomes by TEM. As shown in Fig. 1B, GX15-070 treatment induced autophagosome formation. The number of autophagosomes per cell was significantly higher in the GX15-070-treated cells compared to no drug treatment $(\mathrm{p}<0.0001$, Fig. 1C).

Synergistic antitumor interactions of CQ and GX15-070 in pancreatic cancer cells. Autophagy has been shown to be 

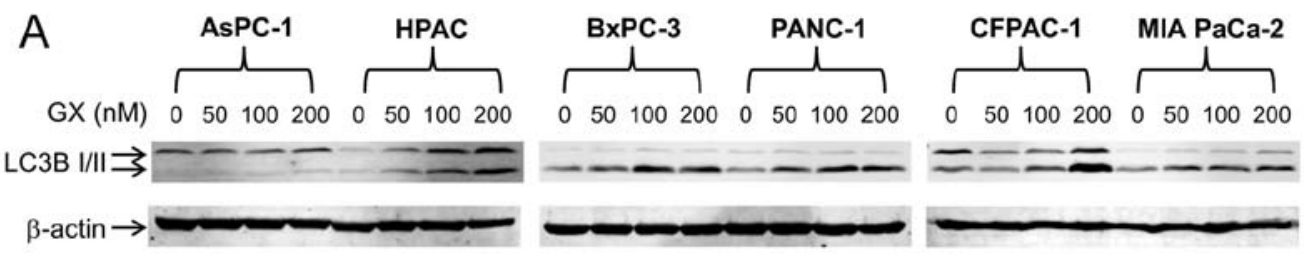

B
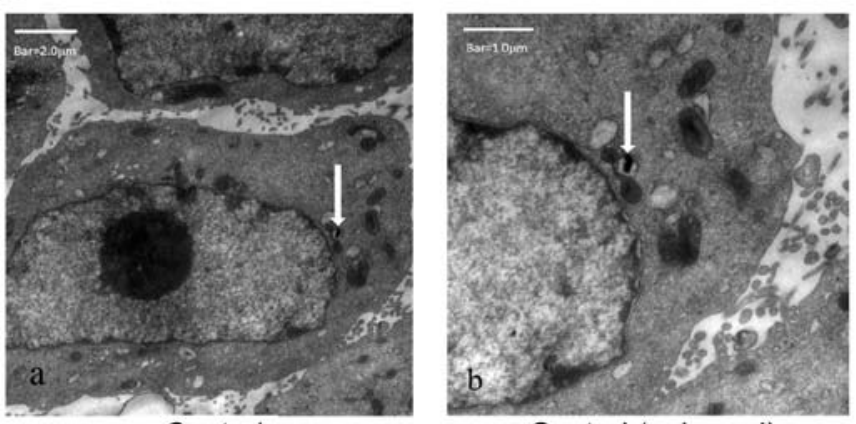

C

Control

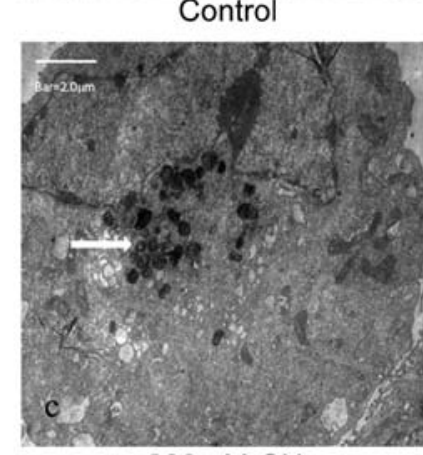

$200 \mathrm{nM}$ GX
Control (enlarged)

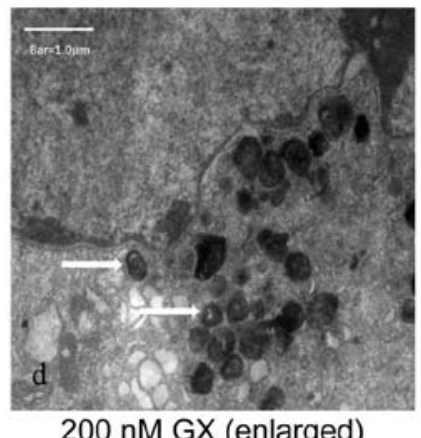

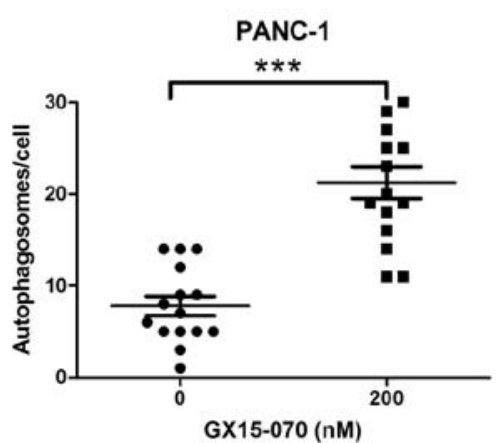

Figure 1. GX15-070 induces autophagy in pancreatic cancer cell lines. (A) AsPC-1, HPAC, BxPC-3, PANC-1, CFPAC-1 and MIA PaCa-2 cells were treated with GX15-070 (GX) for $72 \mathrm{~h}$. Soluble proteins were analyzed by western blot analyses probed with anti-LC3B or - $\beta$-actin antibody. (B) PANC-1 cells were treated without ( $\mathrm{a}$ and b) or with $200 \mathrm{nM} \mathrm{GX15-070} \mathrm{(c} \mathrm{and} \mathrm{d)} \mathrm{for} 72 \mathrm{~h}$ and then harvested for transmission electron microscopy analysis. The arrows indicate autophagosomes. Panels (b and d) are higher magnification of the images in panels (a and c), respectively. (C) Autophagosomes per cell were counted for 15 randomly selected cells from each condition and graphed. The horizontal lines indicate mean in each group and the error bars indicate the standard error. The p-value was calculated using the Mann-Whitney U-test. ${ }^{* * *} \mathrm{p}<0.0001$.

protective when cells encounter stress and without it cells can succumb to such cellular stresses leading to cell death $(11,16)$. Therefore, inhibiting autophagy might inhibit the adaptive response to GX15-070 treatment and enhance apoptosis. To test this, we determined the extent and direction of the antitumor interactions of CQ and GX15-070 in pancreatic cancer cell lines by MTT assays. In all six cell lines tested, we found that the combination resulted in additive to synergistic antitumor interactions, as indicated by the combination index values $<1$ (Fig. 2A-F). CQ $\mathrm{IC}_{50}$ was significantly lower in the presence of GX15-070 (Fig. 2G). The reduction in $\mathrm{IC}_{50}$ ranged from 1.5-fold (BxPC-3) to 13.9-fold (PANC-1).

$C Q$ and GX15-070 cooperatively induce apoptosis in pancreatic cancer cells. We then sought to determine if $\mathrm{CQ}$ and GX15-070 treatment induces apoptosis. As indicated in Fig. 3A-C, treatment with GX15-070 or CQ alone induced minimal apoptosis; however, in the combination, significant increase of apoptotic cells was observed in all the cell lines tested except AsPC-1. This was accompanied by cleavage of PARP, as demonstrated in the HPAC cells (Fig. 3C). Although increase of cleaved PARP was not detectable in the BxPC-3 cells, there was a decrease in the full-length PARP. The lack of detection of the cleaved form might be due to the detection limits of the assay. In addition to inducing apoptosis, combined drug treatment also resulted in decreased levels of Bcl-2, Bcl-xL and Mcl-1 (Fig. 4).

\section{Discussion}

Bcl-2 inhibitors have demonstrated promising anticancer activity in a variety of malignancies, including pancreatic cancer $(4-8,12,13,15)$. In our previous study, we demonstrated that clinically achievable concentrations of GX15-070 induced significant growth arrest in pancreatic cancer cell lines. Notably, they only induced minimal levels of apoptosis (9), suggesting that other mechanisms must exist preventing apoptosis from occurring. In this study, we found that GX15-070 induced autophagy in the pancreatic cancer cell lines, which is in agreement with numerous studies using Bcl-2 inhibitors in various malignancies $(4,12,13,15)$.

Pancreatic cancer cells require autophagy for growth and protection from cytotoxic anticancer drugs $(16,17)$, suggesting that it might be an effective drug target for treating pancre- 
A

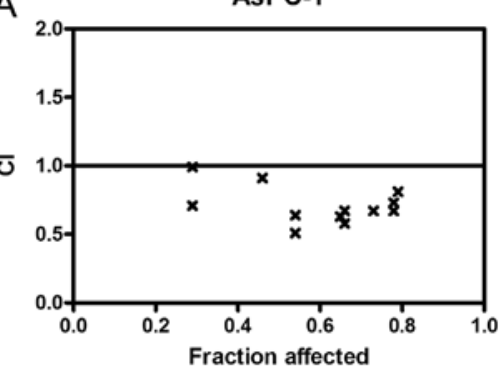

C

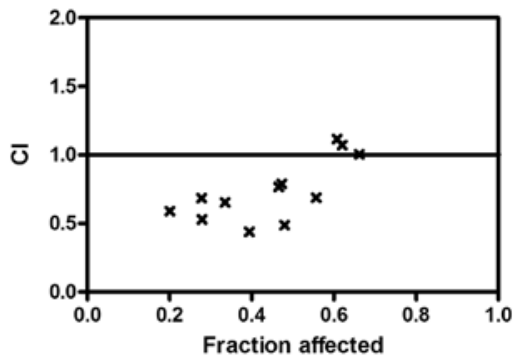

E

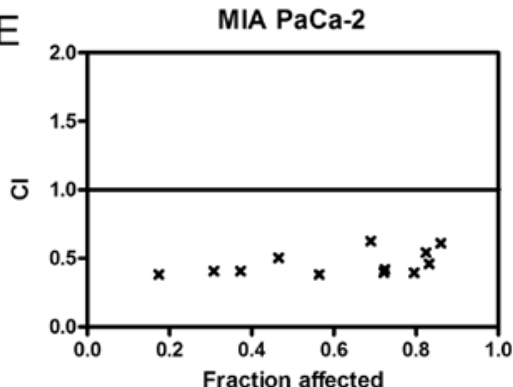

B

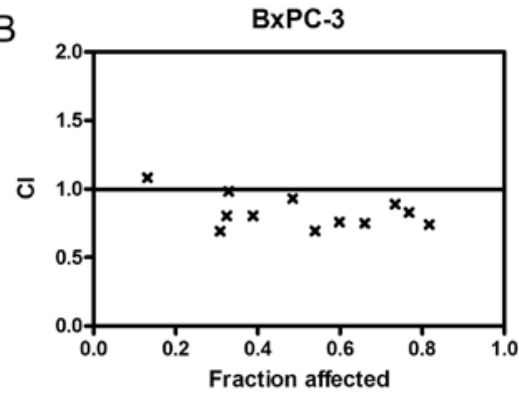

D
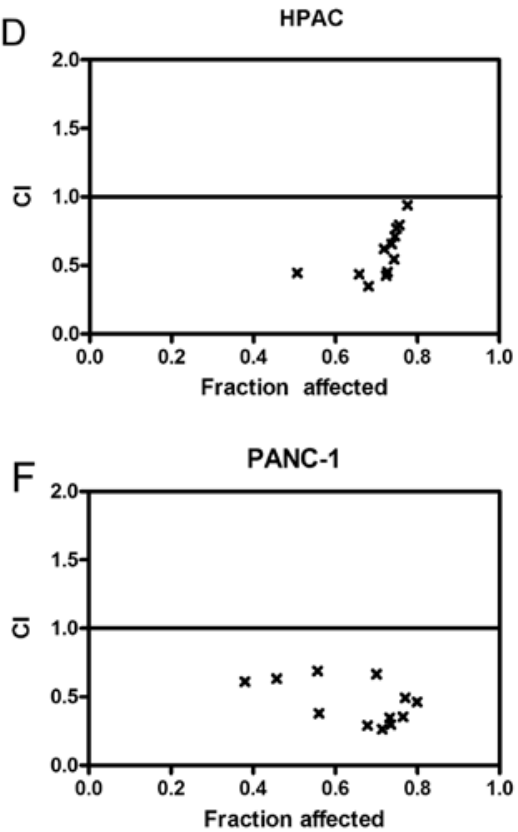

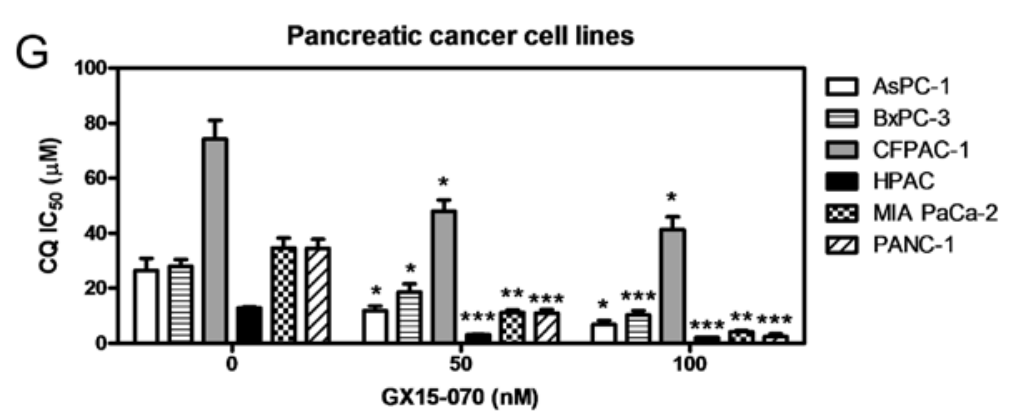

Figure 2. Synergistic antitumor interactions of chloroquine (CQ) and GX15-070 in pancreatic cancer cells. (A-F) Pancreatic cancer cell lines were treated with variable concentrations of CQ for $96 \mathrm{~h}$ in the absence or presence of GX15-070, administered simultaneously. Viable cells were determined by MTT assays. The extent and direction of the antitumor interactions of CQ and GX15-070 were determined using CompuSyn software. The data are presented as combination index vs. fraction affected plots (CI vs. $\mathrm{Fa}$ ). $\mathrm{CI}<1, \mathrm{CI}=1$ and $\mathrm{CI}>1$ indicate synergistic, additive and antagonistic effects, respectively. $(\mathrm{G}) \mathrm{CQ} \mathrm{IC}{ }_{50}$, with or without GX15-070, was calculated using GraphPad Prism 5.0. The data are presented as mean values \pm standard errors from at least three independent experiments. ${ }^{*} \mathrm{p}<0.05,{ }^{* *} \mathrm{p}<0.005$ and ${ }^{* * *} \mathrm{p}<0.0005$, relative to vehicle control.

atic cancer. Recently, the autophagy inhibitor CQ was used in combination with gemcitabine to treat pancreatic cancer cell lines (16). Results of that study found that gemcitabine induced autophagy, which was protective against growth inhibition, and combination with CQ resulted in significant growth inhibition. In line with that study, we demonstrated that GX15-070 induces autophagy and synergizes with CQ to induce growth arrest and enhances apoptosis. In addition to pancreatic cancer, Pan et al demonstrated similar results in esophagus cancer cells indicating that GX15-070 induces cytoprotective autophagy (13).
In summary, in the present study we demonstrated that GX15-070 in combination with CQ may be a promising strategy for treating pancreatic cancer. Our results suggest that GX15-070 induces cytoprotective autophagy which can be overcome by the addition of CQ. CQ has long been used as anti-malarial treatment as well as anti-arthritis treatment and the safety profiles are already established, making it an attractive drug for use in combination with chemotherapy drugs. As the combination of GX15-070 and CQ induced variable levels of apoptosis, it may be advantageous to investigate some of the newer more potent $\mathrm{Bcl}-2$ inhibitors in combination with 
A

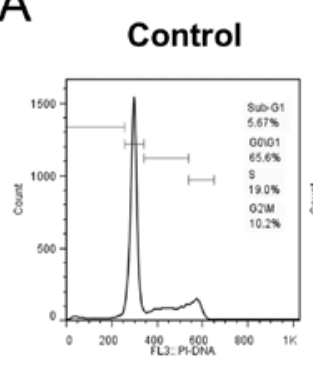

GX $200 \mathrm{nM}$

$\mathrm{CQ} 20 \mu \mathrm{M}$

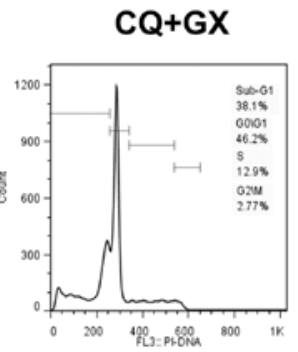

B
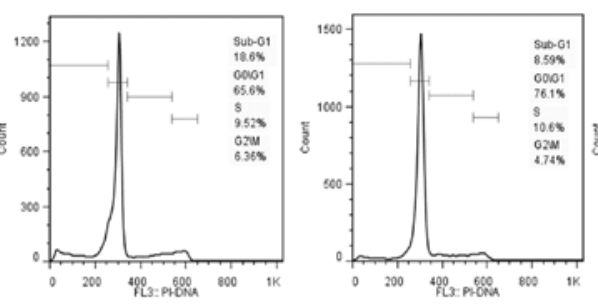

Pancreatic cancer cell lines

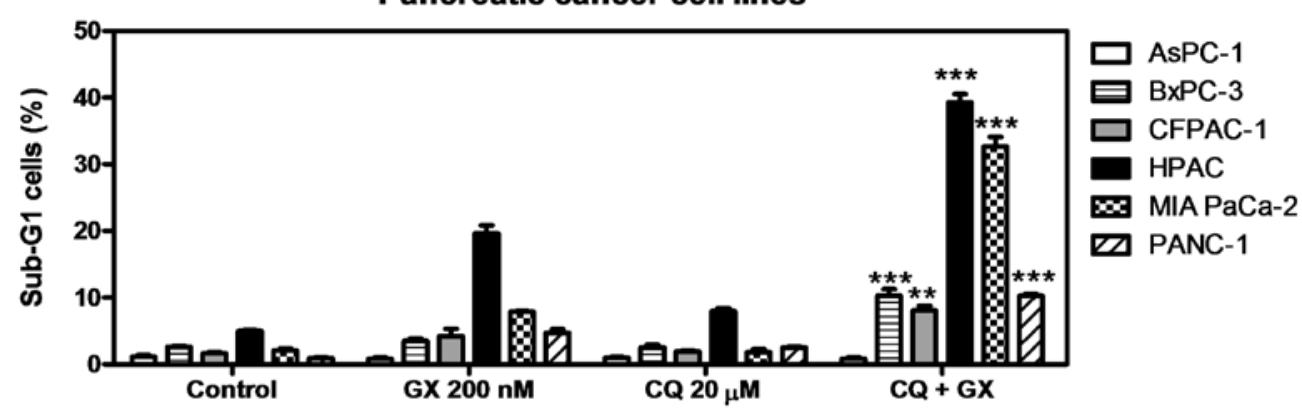

C

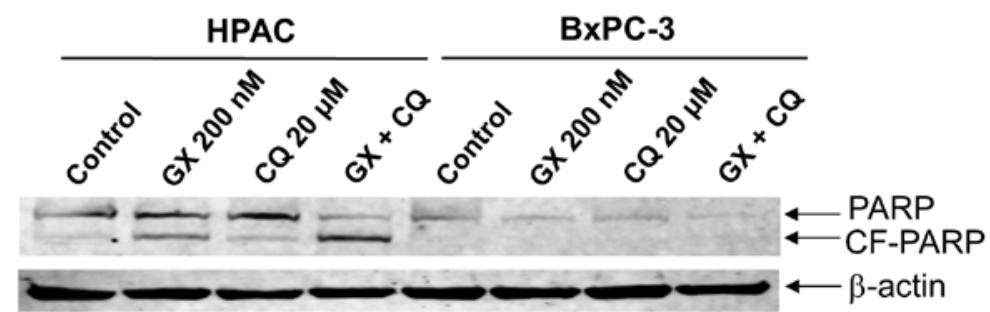

Figure 3. Chloroquine (CQ) enhances GX15-070-induced apoptosis in pancreatic cancer cells. (A) HPAC cells were treated with $20 \mu \mathrm{M} \mathrm{CQ}, 200 \mathrm{nM}$ GX15-070 or in combination for $72 \mathrm{~h}$ and then stained with PI and subjected to flow cytometry analyses. Representative histograms are shown. (B) Apoptotic events were recorded as $\mathrm{PI}^{+}$events with sub-G1 DNA content. The data are presented as mean values \pm standard errors from one representative experiment. (C) HPAC and BxPC-3 cells were treated with CQ, GX15-070 or in combination for $72 \mathrm{~h}$. Whole cell lysates were subjected to western blotting and probed with anti-PARP or $-\beta$-actin antibody. ${ }^{* *} \mathrm{p}<0.005,{ }^{* * *} \mathrm{p}<0.0005$.

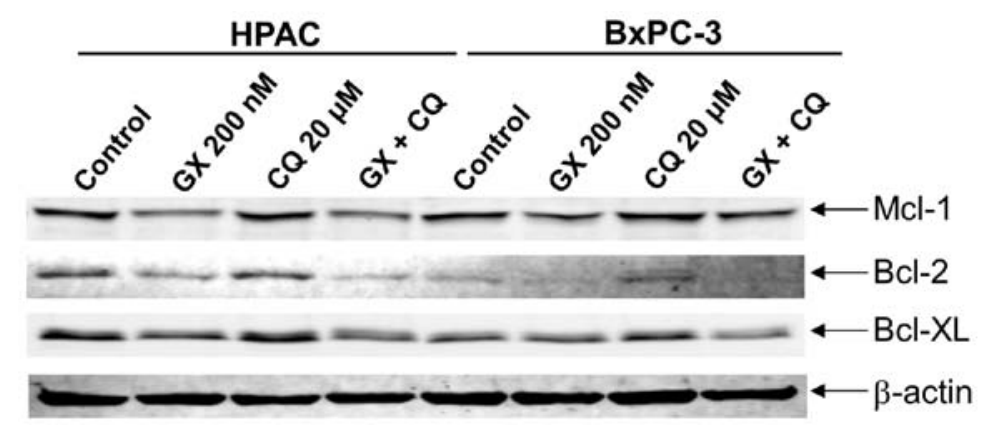

Figure 4. Effects of chloroquine (CQ), GX15-070 or the combination on Bcl-2, Mcl-1 or Bcl-xL expression in pancreatic cancer cell lines. HPAC and BxPC-3 cells were treated with CQ, GX15-070 or in combination for $72 \mathrm{~h}$. Whole cell lysates were subjected to western blotting and probed with anti-Mcl-1, -Bcl-2, -Bcl-xL, or - $\beta$-actin antibody.

CQ. Although our study involved a limited number of cell lines, it provides a compelling rationale for the further study of Bcl-2 inhibitors in combination with CQ in mouse models and holds promise as a potential effective treatment strategy for pancreatic cancer.

\section{Acknowledgements}

This study was funded by a Start-up Fund from Jilin University, Changchun, China, and grants from the National Natural Science Foundation of China (NSFC 31271477 and 81200363). 


\section{References}

1. Siegel R, Ma J, Zou Z and Jemal A: Cancer statistics, 2014. CA Cancer J Clin 64: 9-29, 2014

2. Vincent A, Herman J, Schulick R, Hruban RH and Goggins M: Pancreatic cancer. Lancet 378: 607-620, 2011.

3. Miyamoto Y, Hosotani R, Wada M, et al: Immunohistochemical analysis of $\mathrm{Bcl}-2$, Bax, Bcl-X, and Mcl-1 expression in pancreatic cancers. Oncology 56: 73-82, 1999.

4. Wei Y, Kadia T, Tong W, et al: The combination of a histone deacetylase inhibitor with the Bcl-2 homology domain-3 mimetic GX15-070 has synergistic antileukemia activity by activating both apoptosis and autophagy. Clin Cancer Res 16: 3923-3932, 2010.

5. McCoy F, Hurwitz J, McTavish N, et al: Obatoclax induces Atg7dependent autophagy independent of beclin-1 and BAX/BAK Cell Death Dis 1: e108, 2010.

6. Konopleva M, Watt J, Contractor R, et al: Mechanisms of antileukemic activity of the novel Bcl-2 homology domain-3 mimetic GX15-070 (obatoclax). Cancer Res 68: 3413-3420, 2008.

7. Nguyen M, Marcellus RC, Roulston A, et al: Small molecule obatoclax (GX15-070) antagonizes MCL-1 and overcomes MCL-1-mediated resistance to apoptosis. Proc Natl Acad Sci USA 104: 19512-19517, 2007.

8. Rahmani M, Aust MM, Attkisson E, Williams DC Jr, FerreiraGonzalez A and Grant S: Inhibition of Bcl-2 antiapoptotic members by obatoclax potently enhances sorafenib-induced apoptosis in human myeloid leukemia cells through a Bim-dependent process. Blood 119: 6089-6098, 2012.

9. Chen S, Wang G, Niu X, et al: Combination of AZD2281 (Olaparib) and GX15-070 (Obatoclax) results in synergistic antitumor activities in preclinical models of pancreatic cancer. Cancer Lett 348: 20-28, 2014.

10. Maiuri MC, Zalckvar E, Kimchi A and Kroemer G: Self-eating and self-killing: crosstalk between autophagy and apoptosis. Nat Rev Mol Cell Biol 8: 741-752, 2007.

11. Yang Z and Klionsky DJ: Mammalian autophagy: core molecular machinery and signaling regulation. Curr Opin Cell Biol 22: 124-131, 2010.

12. Malik SA, Orhon I, Morselli E, et al: $\mathrm{BH} 3$ mimetics activate multiple pro-autophagic pathways. Oncogene 30: 3918-3929, 2011.
13. Pan J, Cheng C, Verstovsek S, Chen Q, Jin Y and Cao Q: The BH3-mimetic GX15-070 induces autophagy, potentiates the cytotoxicity of carboplatin and 5-fluorouracil in esophageal carcinoma cells. Cancer Lett 293: 167-174, 2010.

14. Vogler M, Weber K, Dinsdale D, et al: Different forms of cell death induced by putative BCL2 inhibitors. Cell Death Differ 16: 1030-1039, 2009.

15. Maiuri MC, Criollo A, Tasdemir E, et al: $\mathrm{BH} 3$-only proteins and BH3 mimetics induce autophagy by competitively disrupting the interaction between Beclin 1 and $\mathrm{Bcl}-2 / \mathrm{Bcl}-\mathrm{X}(\mathrm{L})$. Autophagy 3: 374-376, 2007

16. Hashimoto D, Blauer M, Hirota M, Ikonen NH, Sand J and Laukkarinen J: Autophagy is needed for the growth of pancreatic adenocarcinoma and has a cytoprotective effect against anticancer drugs. Eur J Cancer 50: 1382-1390, 2014.

17. Yang S, Wang X, Contino G, et al: Pancreatic cancers require autophagy for tumor growth. Genes Dev 25: 717-729, 2011.

18. Edwards H, Xie C, LaFiura KM, et al: RUNX1 regulates phosphoinositide 3-kinase/AKT pathway: role in chemotherapy sensitivity in acute megakaryocytic leukemia. Blood 114: 2744-2752, 2009.

19. Xie C, Edwards H, Xu X, et al: Mechanisms of synergistic antileukemic interactions between valproic acid and cytarabine in pediatric acute myeloid leukemia. Clinical cancer research: an official journal of the American Association for Cancer Research 16: 5499-5510, 2010.

20. Xu X, Xie C, Edwards H, Zhou H, Buck SA and Ge Y: Inhibition of histone deacetylases 1 and 6 enhances cytarabine-induced apoptosis in pediatric acute myeloid leukemia cells. PloS One 6: e17138, 2011.

21. Chou TC: Theoretical basis, experimental design, and computerized simulation of synergism and antagonism in drug combination studies. Pharmacol Rev 58: 621-681, 2006.

22. Wang G, He J, Zhao J, et al: Class I and class II histone deacetylases are potential therapeutic targets for treating pancreatic cancer. PloS One 7: e52095, 2012.

23. Maiuri MC, Le Toumelin G, Criollo A, et al: Functional and physical interaction between $\mathrm{Bcl}-\mathrm{X}(\mathrm{L})$ and a $\mathrm{BH} 3$-like domain in Beclin-1. EMBO J 26: 2527-2539, 2007. 